\title{
Impact of Long-Term Stress on the Light Output of a WRGB AMOLED Display
}

\author{
Frédérique Chesterman, Bastian Piepers, Tom Kimpe, Patrick De Visschere, and Kristiaan Neyts
}

\begin{abstract}
This paper describes how long-term use impacts the light output of a commercial 55" WRGB AMOLED display with InGaZnO TFT backplane. This covers effects which are known by the terms "aging", "image-sticking," and "burn-in." The focus is on three different observations: permanent change in light output as a function of time, permanent screen burn-in, and permanent shift in color point. From this work it can be concluded that state-of-theart OLED displays still suffer from light output instability under prolonged stress. The results suggest that the permanent change in light output can be explained by the combination of three different phenomena: a decrease in efficiency of the OLEDs as a function of time for active subpixels, a positive threshold voltage shift of the driving transistor for active subpixels, and a negative threshold voltage shift of the driving transistor for inactive subpixels, if they are illuminated and/or kept at high temperature. To our knowledge, this is the first work that describes and quantifies the permanent change in light output of a commercial WRGB OLED panel with InGaZnO TFT backplane. It sheds light on which effects occur and can be a valuable tool, both in the design and optimization of OLED panels and in the determining the circumstances under which this technology may be applicable.
\end{abstract}

Index Terms-Aging, AMOLED, burn-in, color shift, image sticking, InGaZnO, instability, WRGB.

\section{INTRODUCTION}

$\mathbf{S}$ INCE more than two decades, Organic Light Emitting Diode (OLED) [1] is considered as a promising technology for application in information displays. The main reason for this is the intrinsic simple structure of an OLED display which is a consequence of the self-emissive nature of the OLED. As each pixel emits its own light there is no need for a backlight and the only remaining components are the substrate, the OLEDs, the encapsulation and the driving electronics, creating the possibility for very thin, light, and theoretically also cheap displays. The self-emissivity of the sub-pixels is also at the root of many other

Manuscript received June 6, 2016; revised October 4, 2016; accepted October 4, 2016. Date of publication October 10, 2016; date of current version January 16, 2017. This work was supported by the Institute for the Promotion of Innovation by Science and Technology in Flanders in the context of the Baekeland Grant "Applicability of OLED technology in medical displays," 130300.

F. Chesterman and B. Piepers are with the Healthcare Division, Barco NV, Kortrijk 8500, Belgium (e-mail: frederique.chesterman@barco.com; bastian.piepers@barco.com).

T. Kimpe is with the Medical Imaging Division, Barco NV, Kortrijk 8500, Belgium (e-mail: tom.kimpe@barco.com).

P. D. Visschere and K. Neyts are with the Department of Electronics and Information Systems, Universiteit Gent, Gent B-9000, Belgium (e-mail: pdv@elis.ugent.be; kneyts@elis.ugent.be).

Color versions of one or more of the figures are available online at http://ieeexplore.ieee.org.

Digital Object Identifier 10.1109/JDT.2016.2615871 advantages of an OLED display, two of which are an infinite contrast and a large viewing angle.

Despite many promising properties in terms of form factor and picture quality, a few problems have so far withheld the wide adoption of OLED technology for large-sized information displays $(>15$ "). The first problem is manufacturing related, namely decreasing yields and consequently increasing production cost when trying to scale up the display size. The second problem is the limited lifetime of an OLED display, resulting from a phenomenon that is widely known by the term "OLED aging" [2]-[4]. The light output of an OLED decreases as a function of its operating time. It is not the decrease as a function of time as such that is the main problem, but the fact that each sub-pixel ages independently from the other sub-pixels, which leads to burn-in. This is in contrast to an LCD and a direct consequence of the self-emitting nature of the OLEDs.

Improving the lifetime of an OLED has been the topic of extensive research over the past years and decades and in 2014, the first OLED panels for TV applications came on the market. This raised the expectations that OLED aging had been solved to the extent that adoption of the technology in consumer applications no longer posed serious problems. However, the applicability of OLED panels for professional applications such as medical imaging [5], [6], with much higher requirements, has not been proven yet. It is the purpose of this paper to assess to what extent and in which form OLED aging is present in state-of-the-art consumer OLED panels, and from that, draw conclusions on the applicability of OLED technology in professional and critical display markets such as medical imaging.

It is important to stress that the focus in this work is on whitered-green-blue (WRGB) active-matrix OLED (AMOLED) and Indium-Gallium-Zinc-Oxide ( $\mathrm{InGaZnO)}$ thin-film-transistor (TFT) backplane (Section II) which are currently the preferred technologies for large-sized display applications. However, WRGB OLED is intrinsically less efficient than red-green-blue (RGB) OLED technology requiring stronger OLED driving to reach a certain light output. It may therefore be that not all of the presented results are representative for displays based on the RGB OLED technology or those using a low temperature poly-silicon (LTPS) TFT backplane, which is currently the standard for small-sized displays.

The remainder of this article is divided in four sections. A first section describes the device under test. A second section focuses on luminance decrease as a function of time. In a third section it is investigated how this results in screen burn-in, and 


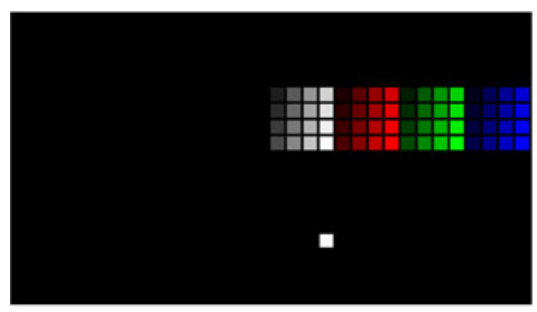

(a)

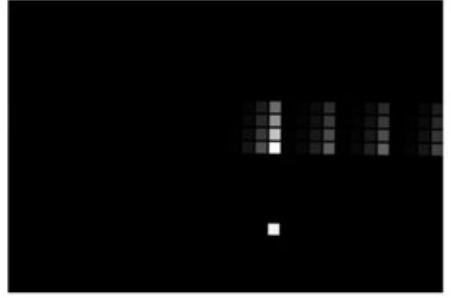

(b)

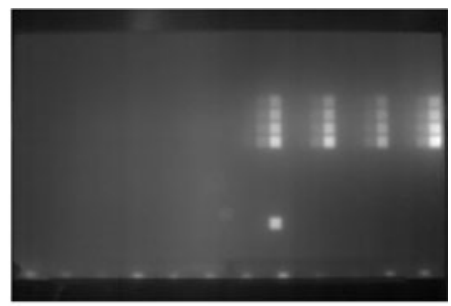

(c)

Fig. 1. (a) Schematic patch applied on the screen of a 55" WRGB OLED display during $1000 \mathrm{~h}$, as part of an aging experiment. The image contains 64 squares, all of which are driven at different Digital Driving Levels (DDLs). The DDLs are of the form $(\mathrm{x}, \mathrm{x}, \mathrm{x}),(\mathrm{x}, 0,0),(0, \mathrm{x}, 0),(0,0, \mathrm{x})$, with $\times$ from 30 to 255 with step 15. (b) Grayscale CCD camera capture of a 55" WRGB OLED display at the start of a $1000 \mathrm{~h}$ aging experiment. (c) IR camera capture of a 55" WRGB OLED display at the start of a $1000 \mathrm{~h}$ aging experiment.

finally, in a fourth section, we look at the impact on the stability of the color point.

\section{DeVICE Under Test}

In this paper, the impact of long-term use on the light output of an OLED display is investigated through measurements on a 55" AMOLED display with an InGaZnO TFT backplane and a resolution of $1920 \times 1080$ pixels. The OLED display uses the WRGB approach meaning that each pixel is made up of four sub-pixels, each comprising a white OLED. Three out of four sub-pixels have a color filter placed in front of them to convert the white light into colored light. The white OLEDs contain a layer emitting blue light and a layer emitting yellow light. The combination of blue and yellow light results in white light.

In the remainder of this article two types of white light, emitted by the display, will often be investigated. On the one hand there is the white light emitted by the white sub-pixel. As there is no filter in front of this sub-pixel, this light corresponds to the light emitted by the white OLED. For the display under study, the RGB Digital Driving Level (DDL) $(252,255,215)$ can be used to drive only the white sub-pixel. On the other hand there is the white light corresponding to the white color point when driving at DDL $(255,255,255)$. In this case, both the white, red and blue sub-pixels are active, though at different intensities.

\section{Luminance DeCREASE AS a Function of Time}

It is well-known that light emission from an OLED reduces as a function of time. This effect is called "OLED aging" [2]-[4]. As each sub-pixel in an OLED display comprises a white OLED it is important to investigate how the emission of light from the display evolves as a function of time. This is the topic of this section.

\section{A. Description of the Experiment}

To investigate the luminance decrease as a function of time, an experiment is set up in which the image in Fig. 1(a) is displayed on the screen of the OLED display during $250 \mathrm{~h}$ continuously. The experiment is repeated four times resulting in a total of $1000 \mathrm{~h}$ of measurement data. The image contains 16 white squares, 16 red squares, 16 green squares and 16 blue squares, each having a size of $50 \times 50$ pixels $(\sim 0.1 \%$ of the display area). The driving levels for each color are DDL 30, 45, 60, $75,90,105,120,135,150,165,180,195,210,225,240$ and

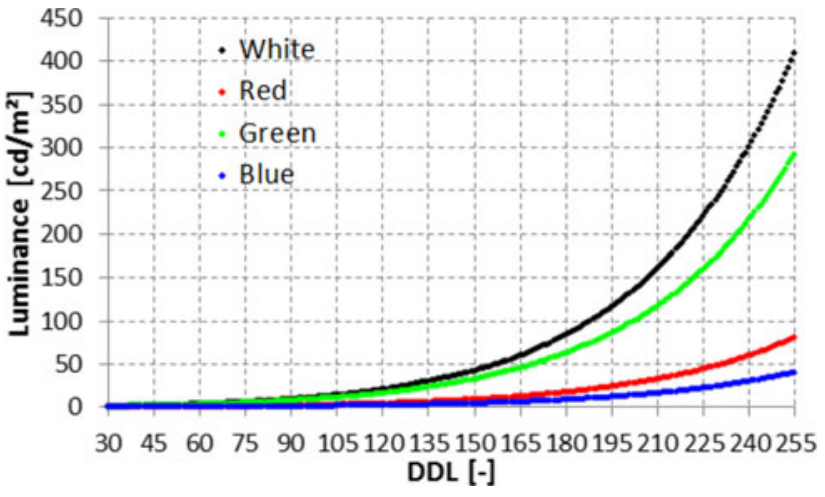

Fig. 2. Luminance as a function of DDL for a 55" WRGB OLED display.

255. It has been found earlier [7] that the rate by which OLED aging takes place is a function of the current density flowing through the OLED. Displaying squares with different colors and DDLs allows investigating OLED aging at different current densities. For each color, the luminance of the display is plotted as a function of the DDL in Fig. 2. From this figure one can read the luminance for each of the 64 squares. This will help the interpretation of the results described below.

The OLED driver uses an algorithm that gradually scales back the display's luminance when static content is displayed. Since we are interested in the aging rate at the display's full performance in terms of brightness, we use a simple trick to avoid this downscaling. Once every $30 \mathrm{~s}$, the left half of the screen is driven at DDL $(255,255,255)$ for one second. This way, the display treats the content as dynamic and the downscaling does not take place.

Throughout the $1000 \mathrm{~h}$ measurement period, the light output of the display is measured by means of a charge-coupled-device (CCD) camera, taking pictures every $10 \mathrm{~min}$. This results in a total of 6000 images of which one is shown in Fig. 1(b). In a CCD camera, impinging light is converted into an electrical signal, according to a specific spectral transfer characteristic. Since this characteristic is not flat as a function of wavelength, the measurements cannot be used to compare the light output for the different colors, without any knowledge on the shape of the sensor's transfer characteristics. However, it does allow looking at relative changes in light output, as long as the shape of the spectrum of the light source remains the same. 


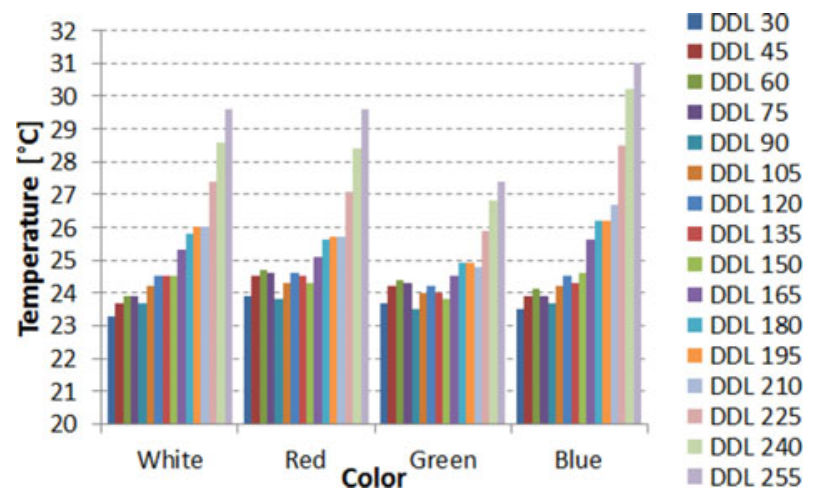

Fig. 3. Temperature of the screen of a 55" WRGB OLED display, in each of the 64 squares [Fig. 1(a)], averaged over $1000 \mathrm{~h}$ of measurements. The temperature is measured by means of an IR camera.

Throughout the $1000 \mathrm{~h}$ period, also the temperature distribution across the OLED display is measured every ten minutes, using an infrared (IR) camera. This results in a total of 6000 images of which one is shown in Fig. 1(c). OLEDs heat considerably as a result of their limited efficiency [8], [9]. Heat is also created in the driving transistors and the power lines. The IR camera image in Fig. 1(c) shows that the temperature hot spots remain quite local, indicating low thermal conductivity of the materials. Including temperature information in this analysis is important as the rate by which OLED aging takes place is also a function of temperature [7]. The experiment was executed at room temperature. The ambient temperature was measured throughout the entire experiment by means of a thermocouple.

Although current density and temperature are two separate parameters determining the aging rate, the current measurement set-up does not allow separating the influence of both parameters. Indeed, as an increase in current density always results in an increase in temperature, the results presented here show the combined effect of current density and temperature.

\section{B. Results}

To investigate the decrease in light emission as a function of time, the measurements of the CCD camera (expressed as counts), are averaged across each of the 64 squares and for each of the 6000 images. By taking a spatial average, the spatial noise of both the camera and the display are reduced. In the remainder of this section the focus will be on the relative decrease in light emission. In this way, a decrease in counts corresponds to a proportional decrease in luminance.

The luminance $L(t)$ is plotted relative to the initial luminance $L(0)$ for white, red, green and blue at the three highest driving levels (DDL 255, DDL 240 and DDL 225) - in Fig. 5. It can be seen that the luminance decreases substantially as a function of time and that the decrease is stronger for higher DDLs. The luminance decrease (after $1000 \mathrm{~h}$ and expressed as percent) is plotted as a function of the initial luminance at DDL 225, 240 and 255 for white, red, green and blue in Fig. 4. This figure shows that the luminance decrease is approximately proportional to the luminance for each color. The relation between luminance and luminance decrease gives valuable insights into the lifetime that can be expected at a certain luminance.

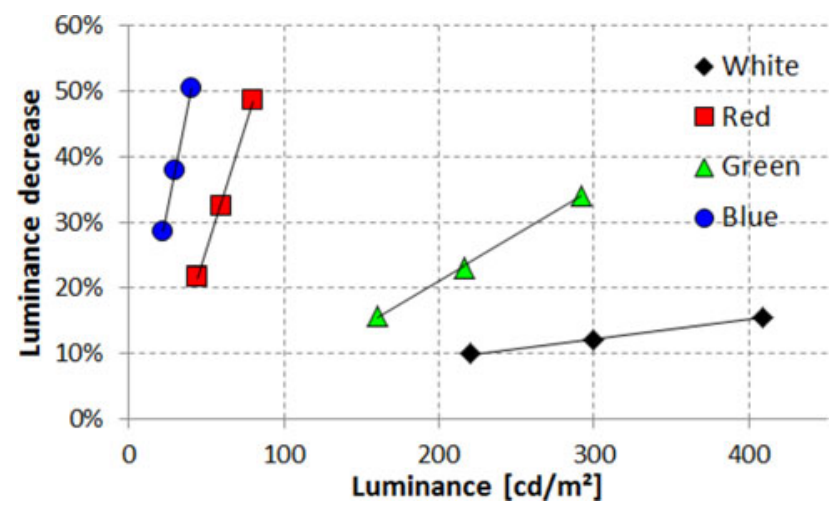

Fig. 4. Luminance reduction after $1000 \mathrm{~h}$ of aging as a function of the initial luminance for white, red, green and blue driven at DDL 225, 240 and 255.

The images acquired with the IR camera are processed in a similar way as the CCD camera images. This allows plotting the temperature of each of the 64 squares as a function of time. The results show that the temperature for each of the squares is relatively stable as a function of time. Therefore, only the average temperature is reported in Fig. 3. The temperature is the highest for blue, followed by white, red and green in decreasing order. A strong relation between DDL and temperature can be observed, but also the position of the square in the pattern plays a role for the temperature.

The power consumption during the aging experiment is $\sim 53 \mathrm{~W}$ of which $\sim 31 \mathrm{~W}$ is consumed by the electronic boards at the back of the display and $\sim 10 \mathrm{~W}$ is consumed by the line drivers when the OLEDs are not driven. The average ambient temperature was $21^{\circ} \mathrm{C}$.

From Fig. 5 it can be seen that the luminance decrease is the largest for blue and red, followed by green and white. As each color is using the same white OLEDs, one would expect a similar behavior for each color. The differences between the color components can be explained by a number of factors:

1) At the same DDL, the white OLEDs are driven at different intensities for red, green and blue. There are two reasons for this: the filter transmission for each color is different and the eye sensitivity for each color is different. Therefore the OLED needs to be driven at a specific intensity (higher for blue and lower for green) to end up with the required light intensity.

2) The pixel area is different for each sub-pixel. Therefore a constant light intensity does not translate into a constant current density for each sub-pixel.

3) The temperature of the OLEDs during the aging experiment is different for each color. This is a direct result of the difference in driving of the pixels.

4) Unlike for red, green and blue, more than one sub-pixel is used when displaying the white color point [DDL (255, $255,255)]$. Since the light is produced by more than one sub-pixel, the average current density is reduced.

\section{SCREEN BURN-IN}

During normal operation, the operating time of pixels in an OLED display is typically not uniform across all pixels. As 


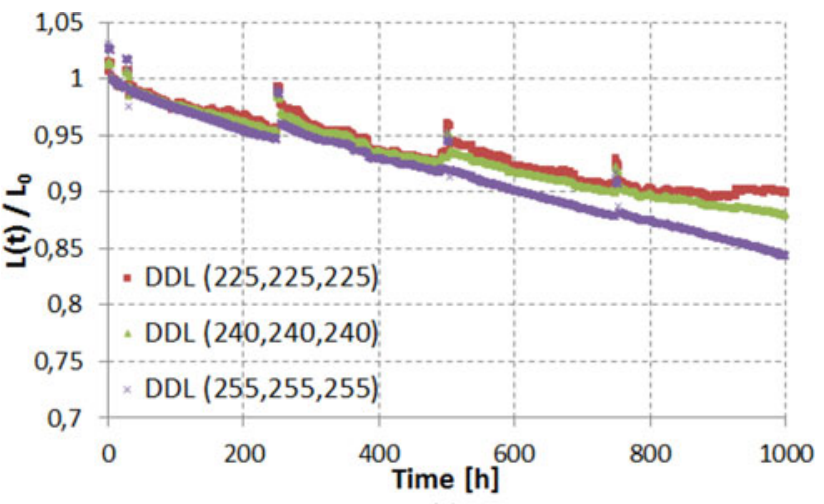

(a)

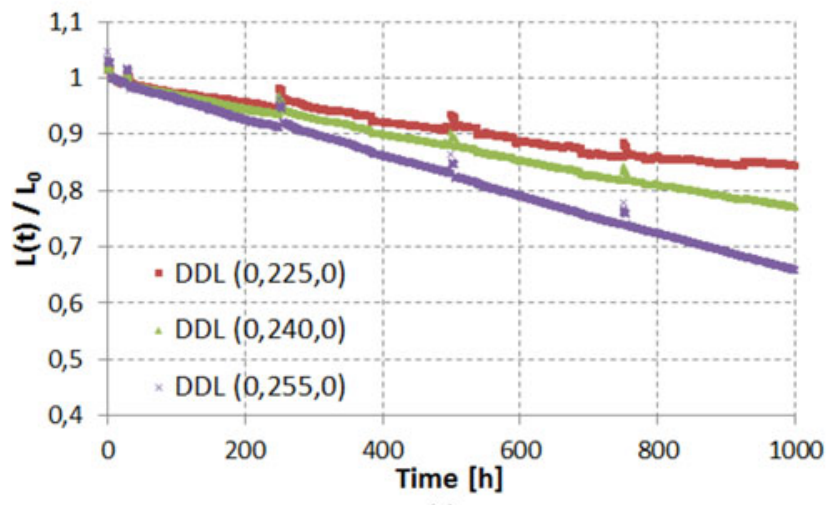

(c)

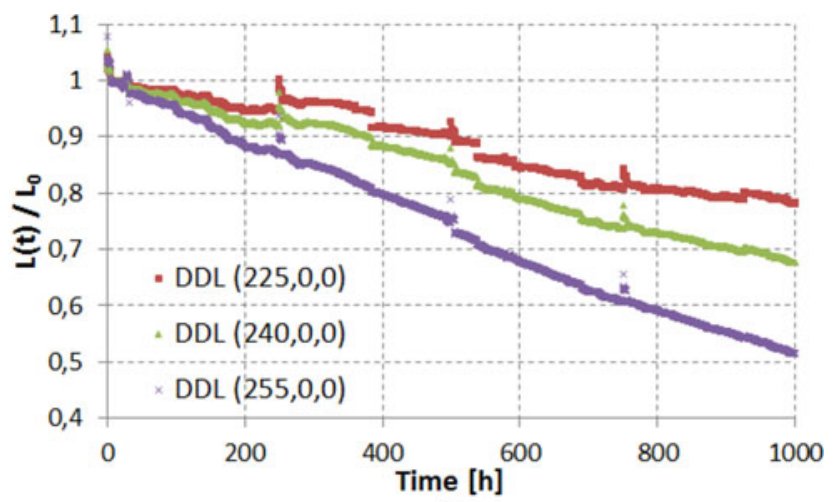

(b)

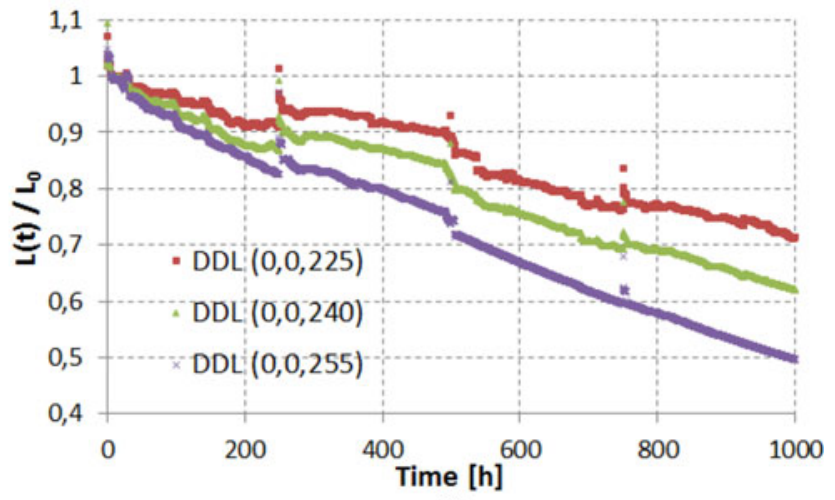

(d)

Fig. 5. Luminance as a function of time during $1000 \mathrm{~h}$, relative to the initial luminance, for white, red, green and blue at four DDLs per color. At DDL $(255,255,255)$, the luminance is reduced by approximately $15 \%$ after $1000 \mathrm{~h}$. At DDL $(255,0,0)$ and $(0,0,255)$, the luminance has reduced by approximately $50 \%$ after $1000 \mathrm{~h}$. At DDL $(0,255,0)$, the luminance has reduced by approximately $15 \%$ after $1000 \mathrm{~h}$. (a) White. (b) Red. (c) Green. (d) Blue.

OLED aging is a function of the operating time of the pixels, this may result in screen burn-in. In this section, the screen burnin on the OLED display after applying the image in Fig. 1(a) for $1000 \mathrm{~h}$ is investigated.

\section{A. Description of the Experiment}

Fig. 1(a) shows the image that was applied during the aging experiment. We are interested in the impact of aging on the light output of the display for each of the 64 squares. To that extent, two experiments are set up.

1) Experiment 1: For this experiment, the four images in Fig. 6, with homogeneous DDL in the area that has been aged, are sequentially displayed on the aged screen. For each patch, a CCD camera picture is taken. The four patches drive the aged zones at DDL $(252,255,215),(149,0,0),(0,157,0)$ or $(0,0,136)$ respectively. As for each of these driving levels, only one sub-pixel (WRGB) is active, this experiment allows investigating the impact of aging for each sub-pixel separately. Moreover, the DDLs for red, green and blue were chosen such that the total power consumption of the panel is the same for each case.

2) Experiment 2: The second experiment investigates the impact of aging on the complete transfer characteristic, i.e. the relation between luminance and DDL. To that extent, a set of images similar to the ones shown in Fig. 6 are sequentially displayed on the aged screen, and for each a CCD camera image is taken. The driving levels of the applied images are of the form
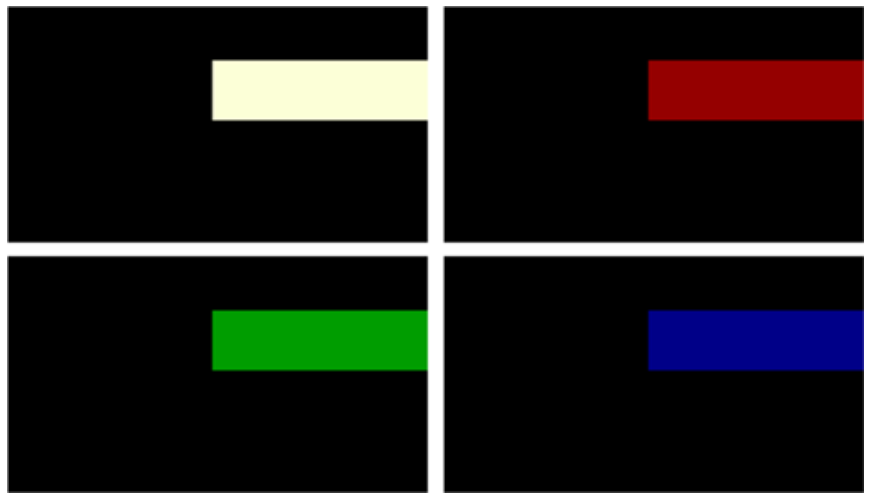

Fig. 6. Patches applied on the screen for investigating the burn-in effect on a WRGB OLED screen after applying the patch in 1 a for $1000 \mathrm{~h}$. For the four DDLs, $(252,255,215),(149,0,0),(0,157,0)$ and $(0,0,136)$, only respectively the white, red, green and blue sub-pixel is driven and the power consumption is equal for all patches. This allows investigating the change in light output for each sub-pixel separately at constant current.

$(\mathrm{x}, 0,0),(0, \mathrm{x}, 0),(0,0, \mathrm{x})$ and $(\mathrm{x}, \mathrm{x}, \mathrm{x})$ with $\times$ between 90 and 255 with step 15.

\section{B. Results}

1) Experiment 1: CCD camera pictures of the screen when applying each of the patches in Fig. 6 are shown in Fig. 7. Upon observation of the CCD camera pictures it is clear that the aging experiment has caused serious burn-in into the screen. 


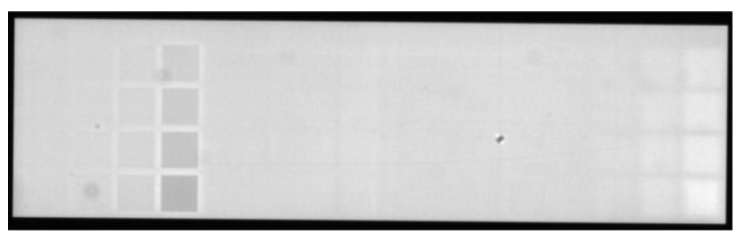

(a)

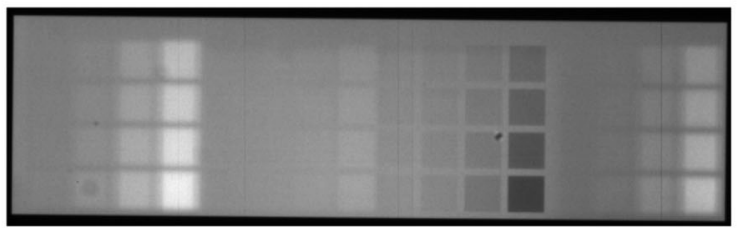

(c)

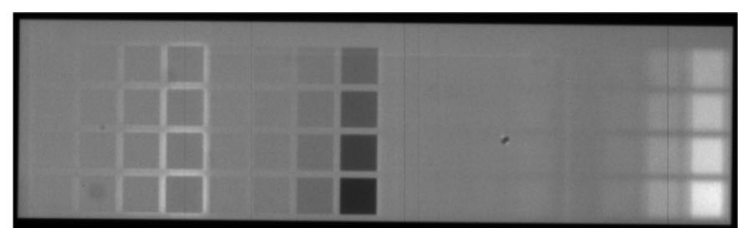

(b)

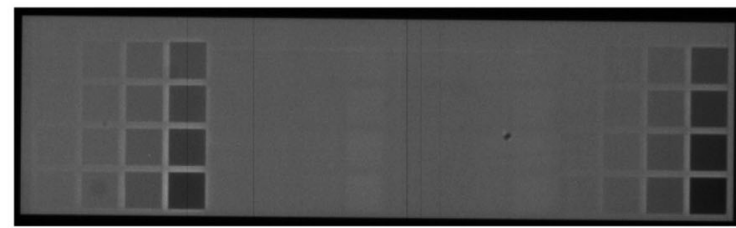

(d)

Fig. 7. CCD gray scale images of part of the 55" WRGB OLED display when applying the images in Fig. 6 after a $1000 \mathrm{~h}$ aging experiment during which the patch in Fig. 1(a) was displayed. Note that the applied images (Fig. 6) are uniform. If the aging experiment had not taken place, the CCD camera would capture a uniform intensity across the rectangle. The captured images are not uniform because the aging pattern caused serious burn-in. For the active pixels, a decrease in light output is observed. For inactive pixels (not driven during the aging experiment), an increase in light output is observed. (a) White. (b) Red. (c) Green. (d) Blue.

Two different types of burn-in can be observed: a decrease in light output with respect to the surrounding pixels and an increase in light output with respect to the surrounding pixels.

A decrease in light output is observed for the regions for which the investigated sub-pixels were active during the aging experiment. This is the case for the first block in Fig. 7(a), the first and second block in Fig. 7(b), the third block in Fig. 7(c) and the first and fourth block in Fig. 7(d). Each time, the burnin effect is more pronounced for the pixels that were driven at higher DDLs during the aging experiment. This decrease in light output is (at least partially) a result of the well-known OLED aging effect [2], [3], [4], [7]. The efficiency of the OLEDs has reduced as a result of prolonged stress during the aging experiment.

An increase in light output is observed for certain regions that were inactive during the aging experiment. This is the case for the fourth block in Fig. 7(a), the fourth block in Fig. 7(b) and the first and fourth block in Fig. 7(c). Also this time, the effect is more pronounced for zones that were driven at high DDLs during the aging experiment. Further, it can be noticed that the effect is the strongest for the zones where a blue square was applied followed by those where a white square was applied. This effect will be studied further in experiment 2 .

2) Experiment 2: In this experiment the full transfer characteristic, i.e. luminance as a function of DDL, is measured before and after the aging experiment. The results are shown in Fig. 8-10. Luminance is determined both by the OLED efficiency and the TFT characteristic. It can be seen that, as a result of the aging experiment, the transfer curve has shifted significantly. In order to determine if this shift is the result of a change in OLED efficiency or a change in TFT characteristic, measuring IV characteristics is required. This was however not possible using the available measurement setup. Still we can draw some conclusions and make some assumptions on the cause of the shifts in Figs. 8-10:

1) The conclusions from experiment 1 , namely the occurence of a decrease in light output for active pixels and an

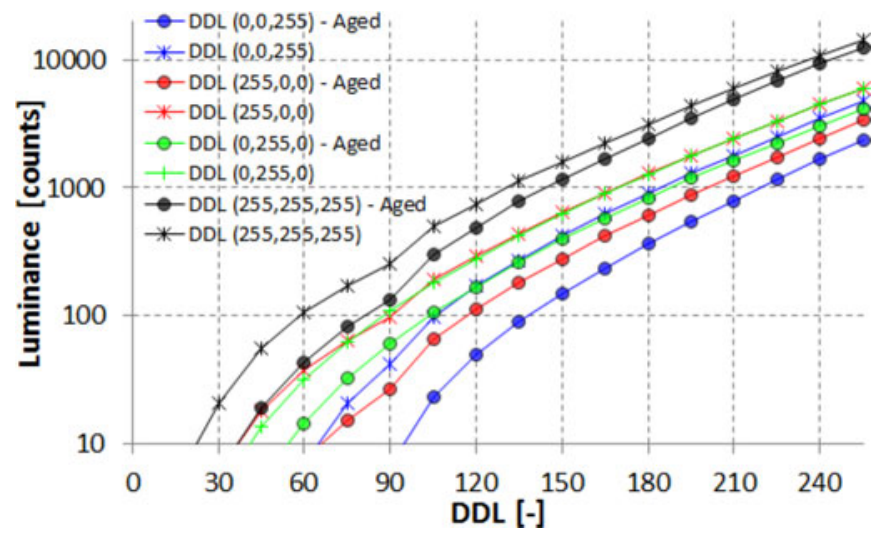

Fig. 8. Luminance level as a function of DDL for DDLs of the form $(\mathrm{x}, \mathrm{x}, \mathrm{x}),(\mathrm{x}, 0,0),(0, \mathrm{x}, 0)$ and $(0,0, \mathrm{x})$ before and after aging for $1000 \mathrm{~h}$ at DDL $(255,255,255),(255,0,0),(0,255,0)$ and $(0,0,255)$ respectively. The investigated sub-pixels were active during the aging experiment. From these results it is suggested that long-term use results in a positive threshold voltage shift combined with a decrease in efficiency for active sub-pixels.

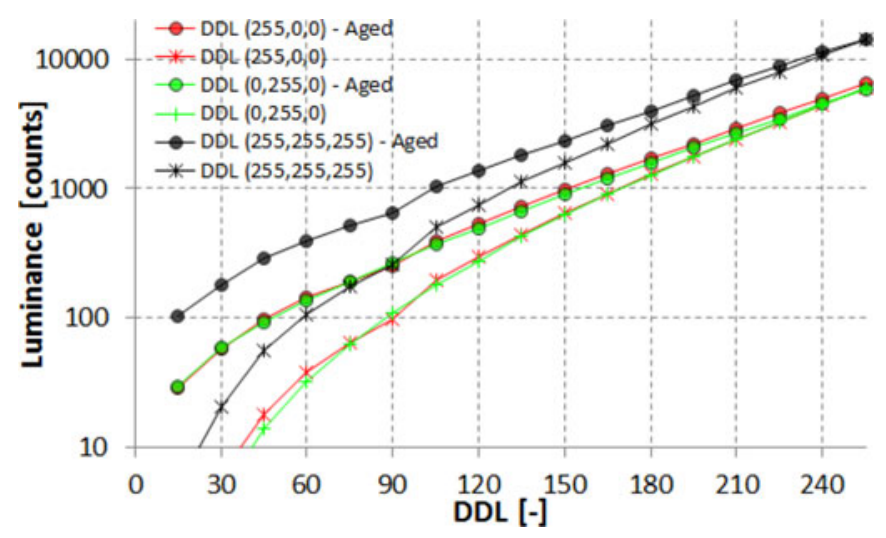

Fig. 9. Luminance level as a function of DDL for DDLs of the form (x, x, $x)$, $(\mathrm{x}, 0,0),(0, \mathrm{x}, 0)$ and $(0,0, \mathrm{x})$ before and after aging for $1000 \mathrm{~h}$ at DDL $(255$, $255,255),(255,0,0),(0,255,0)$ and $(0,0,255)$ respectively. The investigated sub-pixels were inactive during the aging experiment. From these results it is suggested that long-term use results in a negative threshold voltage shift for inactive sub-pixels. 


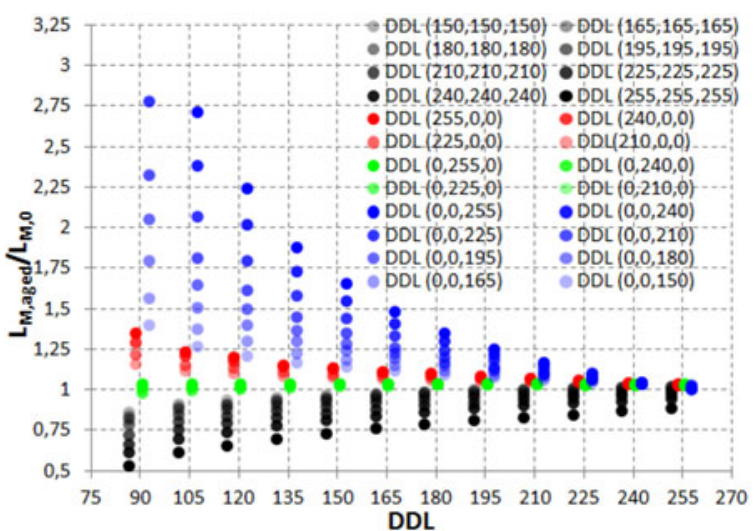

(a)

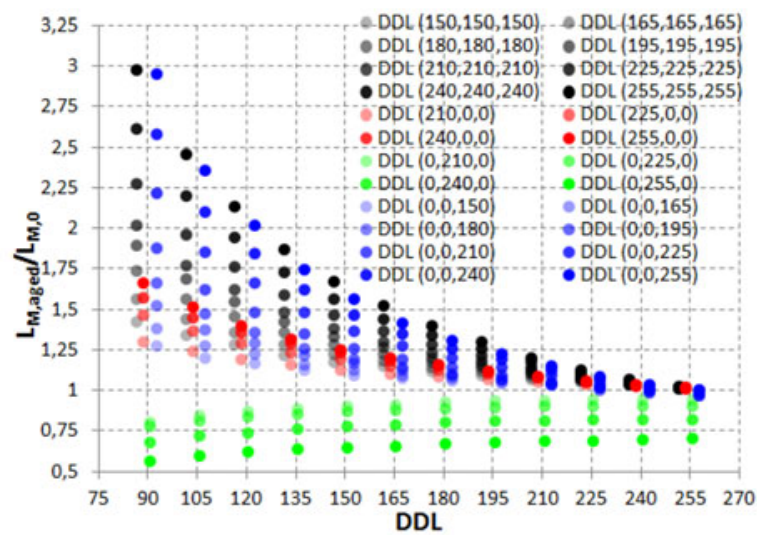

(c)

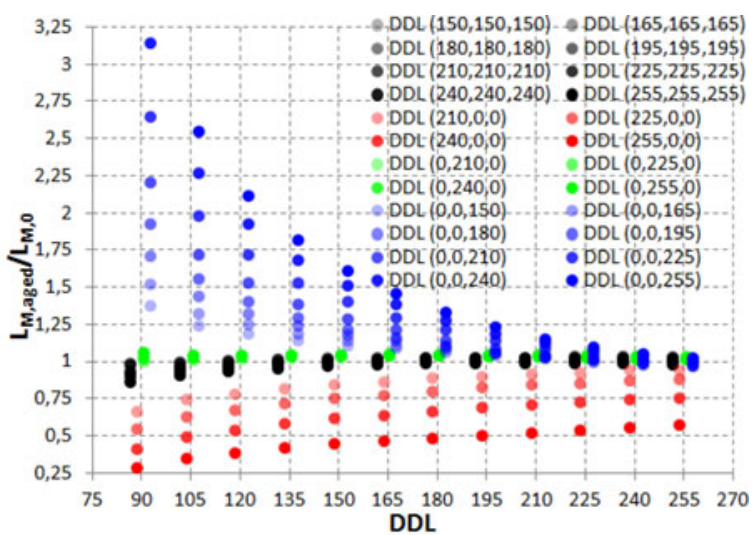

(b)

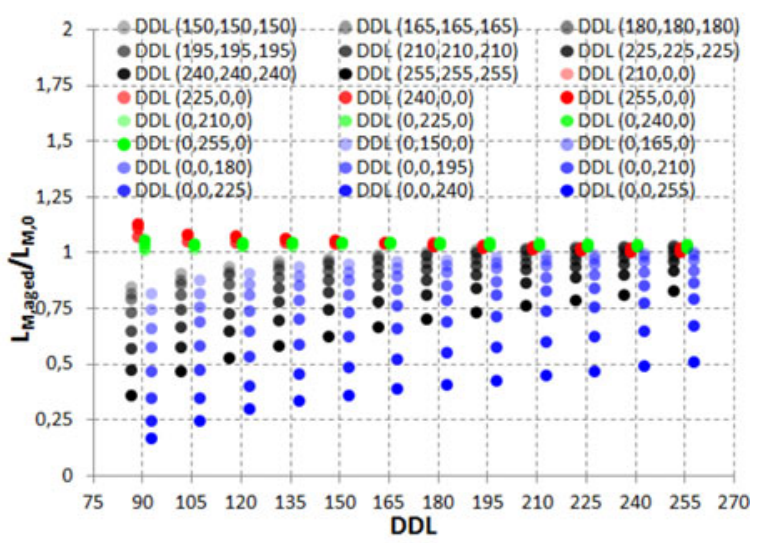

(d)

Fig. 10. Light output after aging / before aging (relative transfer curve) for DDLs of the form (a) (x, $x, x)(b)(x, 0,0),(c)(0, x, 0)$ and (d) $(0,0, x)$ with $\times$ from 90 until 255 with step 15, when aging with the patch shown in Fig. 1(a) for 1000 h. The color of the dots and the legend indicate at which DDL the pixel was driven during the aging experiment. For example in (d) it can be observed that the light output of a blue sub-pixel decreases when a pixel is driven at DDLs of the form $(0,0, x)$ (blue dots) and ( $x, x, x)$ (black dots) for $1000 \mathrm{~h}$ and stays more or less constant when a pixel is driven at DDLs of the form (x, 0, 0) (red dots) and $(0, \mathrm{x}, 0)$ (green dots) for $1000 \mathrm{~h}$. This can be explained by the fact that only DDLs of the form $(0,0, \mathrm{x})$ and $(\mathrm{x}, \mathrm{x}, \mathrm{x})$ are using the blue sub-pixel. In each figure it can be seen that the light output has decreased substantially for pixels that were active during the aging experiment. It is suspected that this is the combined result of a reduction in OLED efficiency ("OLED aging") and a positive threshold voltage shift of the driving TFTs. For the pixels that were inactive, the light output has increased substantially. It is suspected that the increase in light output is a result of a change in threshold voltage of the driving transistor under influence of prolonged illumination. The increase in luminance is the highest for aging with blue DDL $(0,0, \mathrm{x})$. (a) Relative transfer curve (before / after aging) for DDLs of the form ( $\mathrm{x}, \mathrm{X}, \mathrm{x})$, when aging for 1000 hours at 24 different DDLs (indicated in the legend), (b) Relative transfer curve (before / after aging) for DDLs of the form $(\mathrm{x}, 0,0)$ (= change in light output for the red sub-pixel), when aging for 1000 hours at 24 different DDLs (indicated in the legend), (c) Relative transfer curve (before / after aging) for DDLs of the form $(0, \mathrm{x}, 0)$ (= change in light output for the green sub-pixel), when aging for 1000 hours at 24 different DDLs (indicated in the legend), (d) Relative transfer curve (before / after aging) for DDLs of the form $(0,0, \mathrm{x})$ (= change in light output for the blue sub-pixel), when aging for 1000 hours at 24 different DDLs (indicated in the legend).

increase in light output for inactive pixels, are confirmed. This is also visible from Fig. 10.

2) The observation that the relative change in light output is more pronounced for low DDLs than it is for high DDLs (Fig. 10) suggests that the shift in transfer curves can at least partially be explained by a positive shift in threshold voltage of the driving TFT for active pixels (Fig. 8) and a negative shift in threshold voltage of the driving TFT for inactive pixels (Fig. 9).

3) The positive shift in threshold voltage for active pixels needs further investigation but it is suspected that this behavior is a result of bias-stress induced positive threshold voltage shift of the InGaZnO driving TFTs [14]-[17].

4) Also the negative shift in threshold voltage of the driving TFT for inactive pixels needs further investigation, but it is suspected that this behavior is a result of the thermal and photo-instability of the backplane TFTs. Indeed,
InGaZnO based TFTs are known to suffer from threshold voltage instability (negative threshold voltage shift) under prolonged illumination [10]-[12] and heating [13]. Although the sub-pixels for which the light output increased were not active during the aging experiment, they were illuminated by neighboring pixels and kept at a high temperature for a long time.

\section{Discussion}

Based on the experiments described in the previous sections we believe that two phenomena are responsible for the permanent change in light output (image sticking or burn-in) in an OLED display. The first phenomenon is the permanent decrease in efficiency of the OLED under influence of prolonged stress. The rate at which this phenomenon takes place is a function of the current density flowing through the OLED and the temperature of the OLED [7]. The second phenomenon is the 


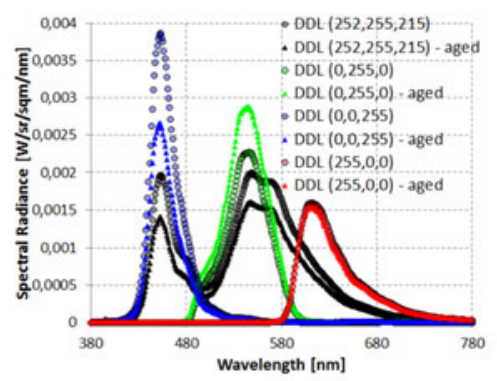

(a)

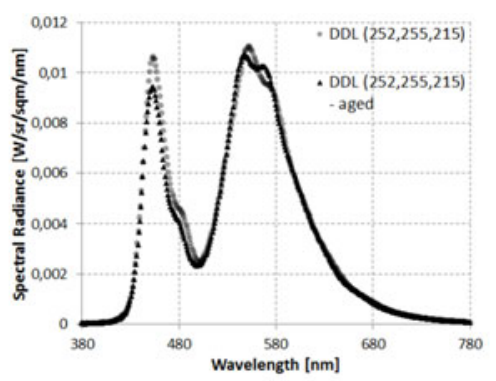

(b)

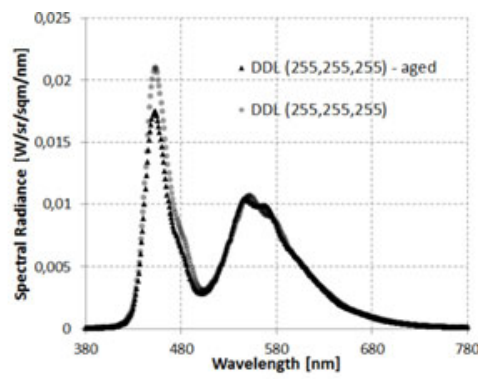

(c)

Fig. 11. Results for the experiments on change in spectrum and shift in color point as a result of long term use of the display. (a) Spectrum for DDL (252,255,215), $(255,0,0),(0,255,0)$ and $(0,0,255)$ before and after DDL $(255,255,255)$ is applied for 1000 hours, (b) Spectrum for the white sub-pixel before and after DDL $(255,255,255)$ is applied for 1000 hours. The spectra are normalized with respect to the power of the yellow peak, (c) Spectrum for DDL $(255,255,255)$ before and after DDL $(255,255,255)$ is applied for 1000 hours. The spectra are normalized with respect to the power of the yellow peak.

shift in threshold voltage of the InGaZnO driving TFT. This is causing a change in light output from the display in two ways. For active pixels there is a positive threshold voltage shift. It results in a decrease in light output which is (in relative terms) most pronounced for low driving levels. The rate at which this phenomenon takes place is function of the current density during stress and possibly also temperature. For inactive pixels there is a negative threshold voltage shift. This is causing an increase in light output which is most pronounced for low driving levels. The rate at which this phenomenon takes place is a function of the intensity of the illumination and possibly also of the temperature. As mentioned before, more specific experiments need to be conducted to confirm the above assumptions.

\section{SHIFT IN COLOR POINT}

The previous experiments describe the relative decrease in light output of each pixel separately without focusing on changes in the color point. Two situations that could lead to a shift in color point can be identified:

1) Each pixel comprises four sub-pixels. For each DDL, the relative contribution of each sub-pixel is calibrated to emit a specific and accurate color point. As the aging rate is a function of the current density flowing through the OLED and the current density flowing through the OLED can be different for each color throughout the lifetime of the display, it can be expected that over time the aging rate will be stronger for one sub-pixel than for another. This results in a distortion of the contribution of each sub-pixel and a corresponding shift in color point.

2) Each sub-pixel comprises a white OLED consisting of a yellow and a blue layer. As both layers are stacked on top of each other, the current flowing through both layers is the same at all time. However, as the materials used in both layers are not equal, it is possible that one layer ages faster than another layer. If this is the case, the color point of the white OLED will shift over time.

In this section it will be investigated if one or both of the above described phenomena take place in our OLED display.

\section{A. Description of the Experiment}

A Yeti spectrometer is used to measure spectra for the square that was aged at DDL $(255,255,255)$ for $1000 \mathrm{~h}$. During the

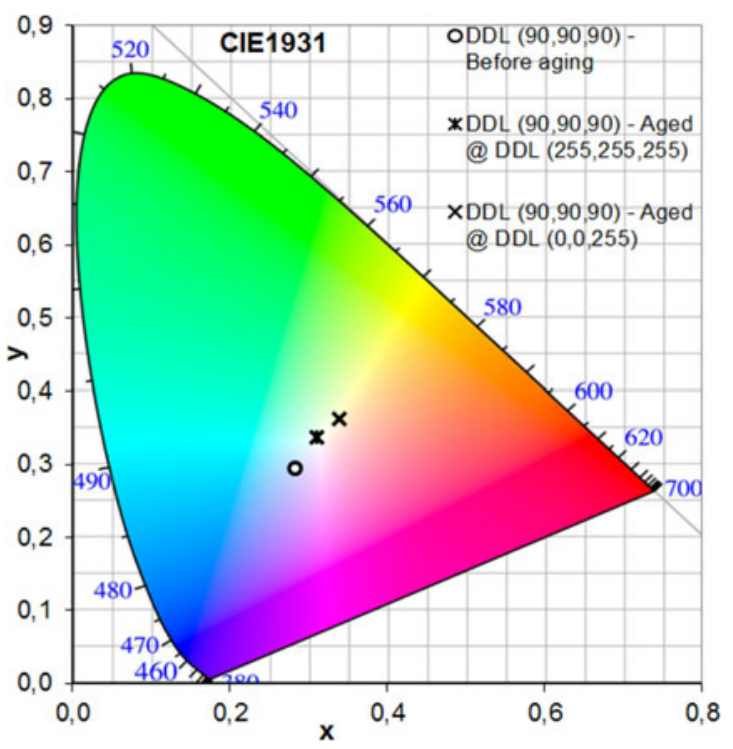

Fig. 12. Color point for DDL $(90,90,90)$ before aging and after aging at $\operatorname{DDL}(255,255,255)$ and $\operatorname{DDL}(0,0,255)$ for $1000 \mathrm{~h}$.

aging experiment, the white and blue sub-pixels, and to a smaller extent also the red sub-pixels, were active. The green sub-pixels were not. The spectra for the white, red, green and blue subpixels are measured by applying a full-screen patch at DDL $(255,0,0),(0,255,0),(0,0,255)$ and $(252,255,215)$. Also the spectrum for DDL $(255,255,255)$ is measured. The obtained spectra are compared to a reference location that has not aged. The results are shown in Fig. 11.

In addition, the color point for DDL $(90,90,90)$ is measured for the zone that was aged at $\operatorname{DDL}(0,0,255)$. We expect a large shift in color point as in this square only the blue sub-pixel was active during the aging experiment. The results are shown in Fig. 12.

\section{B. Results}

The measurements on the square that was aged at DDL $(255,255,255)$ (Fig. 11) show that the light output for green (the inactive sub-pixel) has increased which confirms our previous findings. The shape of the spectrum did not change. The spectrum for red remained largely unchanged which could be 
explained by the small contribution of this sub-pixel when driving at $\operatorname{DDL}(255,255,255)$. The spectrum for blue has decreased substantially as a result of a decrease in OLED efficiency which is also in correspondence with previous findings. It is also interesting to see what happens to the spectrum for DDL $(252,255$, 215) for which only the white sub-pixel is used. As there is no filter in front of this pixel we are measuring the spectrum of the white OLED itself. In Fig. 11(b), the spectrum of the white OLED, before and after applying DDL $(255,255,255)$ for $1000 \mathrm{~h}$, and normalized with respect to the integrated power of the yellow peak, is shown. It can be seen that the shape of the spectrum has changed slightly. The intensity of the blue peak is reduced by $11.5 \%$ with respect to the intensity of the yellow peak, which leads to a shift in color point. Also the shape of the spectrum for DDL $(255,255,255)$ is investigated. The spectrum of the light output for DDL $(255,255,255)$, before and after applying DDL $(255,255,255)$ for $1000 \mathrm{~h}$, and normalized with respect to the power of the yellow peak, is shown in Fig. 11(c). It can be seen from the figure that also for this spectrum the intensity of the blue peak has decreased $(-15.9 \%)$ with respect to the yellow peak, indicating a shift in color point.

In Fig. 12 it can be seen that the color point for DDL $(90,90,90)$ has shifted significantly in the squares that were aged at DDL $(255,255,255)$ and DDL $(0,0,255)$.

From these experiments it can be concluded that two factors contribute to a shift in color point for the OLED display: the change in spectrum of the white OLED resulting from the faster aging rate of the blue layer with respect to the yellow layer and the change in balance of the light output of the different sub-pixels as a result of differences in operating conditions.

\section{CONCLUSION}

In this paper, the results of a number of experiments to characterize the impact of long-term stress on the light output of a WRGB OLED display with InGaZnO TFT backplane, have been presented. The device under test uses the technologies that are currently most mature for large-sized OLED displays. In a first section the luminance reduction was investigated as a function of time. After that, the burn-in effects in the screen were analyzed in detail. Finally, in a last section, the change in spectrum and color point as a result of OLED aging is discussed.

From our measurements it can be concluded that the light output of an OLED display decreases substantially as a function of operating time. The largest decrease is for blue, followed by red, green and white in decreasing order. The rate by which the decrease takes place is roughly proportional to luminance. As sub-pixels are typically driven at different intensities, the balance between the light output of different sub-pixel may change over time and result in a shift in color point. Also the shape of the spectrum of the white OLED may change.

Although aging is a well-known problem for OLED technology, the literature on how it impacts the performance of state-of-the-art OLED displays is scarce. This study has managed to quantify the effect and identified the different com- ponents contributing to the permanent change in light output. It was suggested that the permanent change is not only caused by the decrease in efficiency of the OLED but also by the positive (under current stress) and negative (under illumination and temperature stress) threshold voltage shift of the $\mathrm{InGaZnO}$ driving TFTs.

This work allows drawing conclusions under which circumstances and for which applications OLED technology may or may not be used to prevent severe reduction in picture quality over time. Our results indicate that OLED technology may be suitable for display applications where the content is dynamic and moderate in brightness. Without additional compensation, the display will suffer from burn-in for applications for which the content is mostly static, as certain pixels will be used more often than others.

\section{REFERENCES}

[1] C. W. Tang and S. A. van Slyke, "Organic electroluminescent diodes," Appl. Phys. Lett., vol. 51, no. 12, pp. 913-915, 1987.

[2] F. So and D. Kondakov, "Degradation mechanisms in small-molecule and polymer organic light-emitting diodes," Adv. Mater, vol. 22, no. 34, pp. 3762-3777, 2010.

[3] H. Aziz and Z. D. Popovic, "Degradation phenomena in smallmolecule organic light-emitting devices," Chem. Mater, vol. 16, no. 23 , pp. 4522-4532, 2004.

[4] S. Gardonio et al., "Degradation of organic light-emitting diodes under different environment at high drive conditions," Organ. Electron., vol. 8, no. 1, pp. 37-43, 2007.

[5] N. Odlum et al., "Preliminary display comparison for dental diagnostic applications," Proc. SPIE, vol. 8318, no. 83181S, 2012.

[6] T. Kimpe and C. Marchessoux, "Important differences between medical displays and normal desktop displays and underlying reasons," Americas Display Eng. Appl. Conf. (ADEAC), Atlanta, GA, USA, 2006.

[7] M. Ishii and Y. Taga, "Influence of temperature and drive current on degradation mechanisms in organic light-emitting diodes," Appl. Phys. Lett., vol. 80, no. 18, 2002, p. 3430.

[8] F. Chesterman et al., "Power consumption and temperature distribution in WRGB active-matrix OLED displays," J. Display Technol., vol. 12, no. 6, pp. 616-625, 2016.

[9] F. Chesterman et al., "Temperature distribution in WRGB activematrix OLED displays," SID Symp. Dig. Techn. Papers, vol. 47, no. 1, pp. 1764-1767, 2016.

[10] J. Jeon, J. Kim, and M. Ryu, "Instability of an amorphous indium gallium zinc oxide TFT under bias and light illumination," J. Korean Phys. Soc., vol. 58, no. 1, pp. 158-162, 2011.

[11] K. Lee et al., "The effect of moisture on the photon-enhanced negative bias thermal instability in Ga-In-Zn-O thin film transistors," Appl. Phys. Lett., vol. 95, no. 23, 2009, p. 232106.

[12] K. H. Ji et al., "Comprehensive studies of the degradation mechanisms in amorphous InGaZnO transistors by the negative bias illumination stress," Microelectron. Eng., vol. 88, no. 7, pp. 1412-1416, 2011.

[13] G. Chang et al., "Temperature-dependent instability of bias stress in InGaZnO thin-film transistors," IEEE Trans. Electron Devices, vol. 61, no. 6, pp. 2110-2124, Jun. 2014.

[14] A. Suresh and J. F. Muth, "Bias stress stability of indium gallium zinc oxide channel based transparent thin film transistors," Appl. Phys. Lett., vol. 92 , no. 3, 2008, p. 033502.

[15] J. Lee, I. Cho, J. Lee, and H. Kwon, "Bias-stress-induced stretchedexponential time dependence of threshold voltage shift in InGaZnO thin film transistors," Appl. Phys. Lett., vol. 93, no. 9, 2008, p. 093504.

[16] K. Hoshino, D. Hong, H. Chiang, and J. Wager, "Constant-voltage-bias stress testing of a-IGZO thin-film transistors," IEEE Trans. Electron Devices, vol. 56, no. 7, pp. 1365-1370, Aug. 2009.

[17] E. Cho et al., "Analysis of bias stress instability in amorphous InGaZnO thin-film transistors," IEEE Trans. Device Mater. Reliability, vol. 11, no. 1, pp. 112-117, Mar. 2011. 


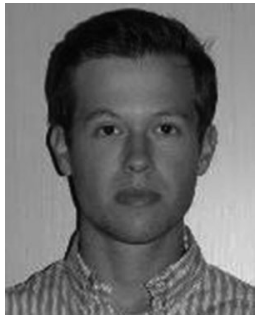

Frédérique Chesterman received the B.S. and M.S. degrees in electrical engineering from Ghent University, Ghent, Belgium, in 2011 and 2013, respectively. $\mathrm{He}$ is currently working toward the Ph.D. degree in photonics engineering in the Electronics and Information Systems, Department of the Faculty of Engineering Sciences and Architecture, Ghent University, Belgium.

Since 2013, he has been a Research Engineer in the Technology and Innovation Group, Barco Healthcare, Kortrijk, Belgium. His research interests include characterization and modeling of OLED displays and development of compensation algorithms, with specific focus on the medical display market.

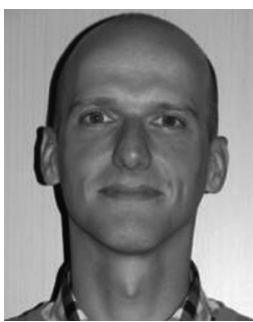

Bastian Piepers received the M.S. degree in electrical engineering from Katholieke Universiteit Leuven, Leuven, Belgium, in 2001.

After his graduation, he joined Philips Innovative Applications as Hardware Engineer for developing high end television sets. Afterwards he was with Televic where he first participated to multiple research projects and became later the architect and project lead for the development of nurse call systems. He is currently a Project Manager of the Technology and Innovation Group, Barco Healthcare, Kortrijk,

Belgium.

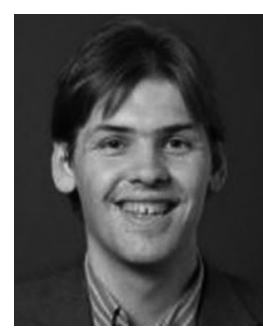

Tom Kimpe received the M.S. degree in computer engineering in 2001 and the Ph.D. in electrical engineering from from Ghent University, Ghent, Belgium, on the topic of image quality of medical displays. In 2010, he finalized a Master in Business Administration degree at the Vlerick Management School.

Since 2001, he has been working in Barco's Healthcare Division, Kortrijk, Belgium. He has taken the positions of Development Engineer, Project Manager, Innovation Manager, the VP of Technology and Innovation, and the Chief Technology Officer of Barco's Healthcare Division. $\mathrm{He}$ is coordinating all research and innovation activities of Barco's Healthcare Division worldwide and is steering an international team of engineers in USA, Belgium, and Italy. His main topics of expertise are (medical) display technology, image and signal processing, image quality modelling and human perception, network technology, and medical regulatory aspects.

Dr. Kimpe is an acknowledged international expert, Member of several program committees (e.g., SPIE medical imaging, SID) and Reviewer for various journals. He also was one of the organizers of the MIPS2015 Medical Imaging Perception Society Conference.

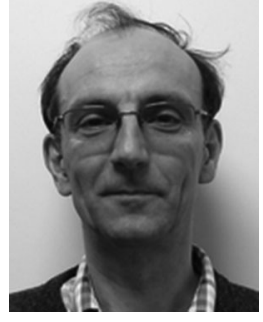

Patrick De Visschere received the degree in electrical engineering in 1976 and the Doctoral degree in applied sciences in 1981, both from the Ghent University, Ghent, Belgium.

In 1976, he joined the Department of Electronics and Information Systems, Ghent University, where he has been working on thin-film solar cells. From 1984 to 2000 , he was affiliated with IMEC, and did research on thin-film electroluminescence. In 1993, he became part-time, and in 2000 full-time, Associate Professor in physical electronics and visualisation technology at the Ghent University. His main research interests include physical electronics and optics, electromagnetism, and ray tracing

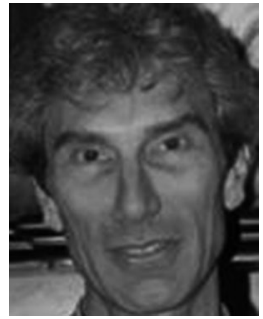

Kristiaan Neyts receicved the Ph.D. degree in 1992 from Ghent University on thin film electroluminescence and made a postdoc at UC Berkeley in 1997-1998.

$\mathrm{He}$ is a full Research Professor in the Electronics and Information Systems, Department of the Faculty of Engineering Sciences and Architecture, Ghent University, Ghent, Belgium. He is currently heading the Liquid Crystals and Photonics Group that is conducting research in the fields of liquid crystals, OLEDs, electrophoresis, and microscopy. This group of 20 researchers has expertise in device technology, numerical simulations, and electro-optical characterization of photonic components. Since 2004, he has been the promoter of 18 completed Ph.Ds. 